\title{
Genetic Screening of the Mitochondrial Rho GTPases MIRO1 and MIRO2 in Parkinson's Disease
}

\author{
Anna Anvret ${ }^{1}$, Caroline Ran ${ }^{1}$, Marie Westerlund ${ }^{1,2}$, Olof Sydow ${ }^{3}$, Thomas Willows ${ }^{3}$, Lars Olson ${ }^{1}$, \\ Dagmar Galter ${ }^{1}$ and Andrea Carmine Belin*,1 \\ ${ }^{1}$ Department of Neuroscience, Karolinska Institutet, Stockholm, Sweden \\ ${ }^{2}$ Present address: Department of Neurobiology, Care Sciences and Society, Karolinska Institutet, Stockholm, Sweden \\ ${ }^{3}$ Department of Neurology, Karolinska University Hospital, Stockholm, Sweden
}

\begin{abstract}
MIRO1 and MIRO2 (mitochondrial Ras homolog gene family, member T1 and T2) also referred to as RHOT1 and RHOT2, belong to the mitochondrial Rho GTPase family and are involved in axonal transport of mitochondria in neurons. Because mitochondrial dysfunction is strongly implicated in Parkinson's disease (PD), MIRO1 and MIRO2 can be considered as new candidate genes for PD. We analyzed two non-synonymous polymorphisms and one synonymous polymorphism in MIRO1 and two non-synonymous polymorphisms in MIRO2, in a Swedish Parkinson case-control material consisting of 241 patients and 307 neurologically healthy controls. None of the analyzed polymorphisms in MIRO1 and MIRO2 were significantly associated with PD. Although we did not find a significant association with PD in our Swedish case-control material, we cannot exclude these Rho GTPases as candidate genes for PD or other neurodegenerative disorders.
\end{abstract}

Keywords: Association, mitochondria, single nucleotide polymorphism.

\section{INTRODUCTION}

Degeneration of dopamine (DA) neurons in substantia nigra (SN) pars compacta causes the typical motor symptoms seen in patients with Parkinson's disease (PD); resting tremor, rigidity, bradykinesia and postural instability [1,2]. However, neuropathology in PD is widespread, affecting neurons in areas from the gastrointestinal tract to cerebral cortex [3]. In accordance with the systemic nature of the disease, there is increasing evidence that mitochondrial dysfunction may underlie some forms of PD [4-6]. Mitochondria are essential in all eukaryotic cells for generating ATP, calcium buffering and involvement in programmed cell death [7-9]. To adapt to cellular demands mitochondria undergo fusion and fission, two opposing processes that exist in equilibrium [10]. The mitochondrial network is very dynamic in neurons $[11,12]$ and the rate of mitochondrial fusion and fission is high [13]. Recruitment of mitochondria to specific neuronal compartments has been shown to be an active ATP consuming process [14]. In support of a mitochondrial involvement in $\mathrm{PD}$, the metabolite of 1-methyl-4-phenyl1,2,3,6-tetrahydropyridine (MPTP), which is 1-methyl-4phenylpyridinium $\left(\mathrm{MPP}^{+}\right)$, is a neurotoxin and inhibits the mitochondrial respiratory chain complex I, which causes degeneration of DA neurons in SN [15]. Another inhibitor of complex I is the pesticide rotenone. In rodents it causes selective degeneration of DA neurons, as well as other neurons to a lesser extent, thereby resembling the pathology of $\mathrm{PD}$

*Address correspondence to this author at the Department of Neuroscience, Karolinska Institutet, 17177 Stockholm, Sweden; Tel: +46-8-524 870 51;

Fax: +46-8-323 742; E-mail: andrea.carmine.belin@ki.se
[16]. Complex I activity has been found to be reduced in SN and in platelets from PD patients [17].

Different polymorphisms in mtDNA have been reported to be associated with both increased and decreased risk of PD [18]. We and others have found associations between PD risk and two nuclear genes involved in mitochondrial maintenance, MTIF3 (mitochondrial translation initiation factor 3) and POLG1 (DNA polymerase gamma 1). MTIF3 is part of the initiation complex formation on the mitochondrial $55 \mathrm{~S}$ ribosome and regulates translation of proteins within mitochondria $[19,20]$. A synonymous single nucleotide polymorphism (SNP) in MTIF3, rs7669, has been reported to be associated with PD in cohorts with different geographical origin [21-23]. POLG1 is important for replication and repair of the mitochondrial genome [24]. Variations in length of the polyglutamine tract of $P O L G 1$ have been found to associate with PD by a number of groups in different geographical regions [25-27].

Several of the PARK genes identified by linkage studies have been reported to influence mitochondria in different ways. Mutations in PTEN induced putative kinase 1 (PINK1) at PARK6, reported to regulate mitochondrial fission, cause early-onset familial PD [28]. Mutations in Parkin at PARK2 have been reported to cause autosomal recessive early-onset Parkinsonism [29]. Parkin acts in a similar way as PINK1 [30] and is believed to be recruited from the cytoplasm by PINK1 to initiate autophagic degradation of impaired mitochondria [31]. DJ-1 at PARK7 causes autosomal recessive early-onset Parkinsonism [32] and is suggested to operate in a parallel pathway to that of PINK1/Parkin to maintain mitochondrial function in oxidative environments [33]. Pink1 has 
been shown to be part of a mitochondrial multi-protein complex together with the atypical GTPases Miro1 and Miro2 (mitochondrial Ras homolog gene family, member T1 and T2) and the adaptor protein Milton [34]. This suggests that Pink1 also plays a role in mitochondrial trafficking.

Miro1 and Miro2, also referred to as Rhot1 and Rhot2, belong to the mitochondrial Rho GTPase family and are involved in axonal transport of mitochondria [35,36]. Miro1 and Miro2 were first identified in yeast [37] and later described to share similarities with Rho GTPases [38]. Miro proteins have a C-terminal domain locating them to the mitochondrial outer membrane, as well as two GTPase domains and two calcium binding EF-hands [36,37]. Calcium binding to the EF-hands regulates the trafficking of mitochondria along microtubules $[39,40]$.

Based on the finding that mitochondrial dysfunction has been implicated in PD [4,41], the importance of Mirol and Miro2 in mitochondrial transport $[35,36]$ and the link between Miro and Pink1 [34], we analyzed SNPs in MIRO1 and MIRO2 with regard to PD. We hypothesized that genetic variations in functionally important regions of these two genes might lead to disturbed mitochondrial trafficking, fusion and/or fission disturbances in neurons and hence increase the risk of neurodegenerative events, such as those observed in PD. We therefore investigated the possible association of five SNPs located in functional regions of the proteins MIRO1 and MIRO2. The SNPs were selected from the NCBI database and screened in a Swedish Parkinson casecontrol material (see Table 1). Three SNPs were selected in MIRO1, one synonymous SNP in the GTPase domain (rs16967164) and two non-synonymous SNPs in each of the two EF-hands (rs28630420, rs34538349). The other two SNPs were non-synonymous SNPs located in one of the EFhand domains of MIRO2 (rs1139897 and rs3743912).

\section{MATERIALS AND METHODOLOGY}

\subsection{Subjects}

A Swedish PD case-control material of Caucasian origin was genotyped for three SNPs in MIRO1 and two in MIRO2 (see Table 1). The PD material consisted of 241 individuals from the Stockholm area (mean age 67.0 years, $60.9 \%$ men). The DNA was obtained after informed oral and written con- sent and approval of the local ethics committee, Regionala etikprövningsnämnden, Stockholm, Sweden. All PD subjects met the United Kingdom Parkinson's Disease Society Brain Bank Criteria for PD except that more than one affected first, second or third degree relative was allowed [42]. Control subjects consisted of 307 neurologically healthy spouses of PD patients and individuals from the SNAC-K project (The Swedish National Study on Aging and Care in Kungsholmen) from the Stockholm area (mean age 64.2 years, 40.4\% men). DNA was extracted from blood according to standard protocols.

\subsection{Genotyping}

The SNPs were genotyped with predesigned TaqMan SNP Genotyping Assays: C_1630552_10 (rs16967164), C_60583023_10 (rs28630420), - C_25937296_10 (rs3743912), C_2463996_1_(rs1139897) and a custom designed assay (rs34538349) using a fast real-time PCR instrument (ABI 7500 FAST Real-Time PCR, Applied Biosystems, Foster City, CA, USA). The TaqMan assay contained primers and 5 ' fluorescently labeled (FAM and VIC) minor groove binding probes $(20 \times)$ for detection of the SNPs. Allelic discrimination was run with pre- and custom-designed primers and probes, genotyping master mix $\left(\operatorname{TaqMan}^{\mathbb{B}}, \mathrm{Ap}-\right.$ plied Biosystems, Foster City, CA, USA) and 10-20 ng of genomic DNA in a total reaction volume of $10 \mu \mathrm{l}$ mixed in transparent 96-well plates. The polymerase chain reaction (PCR) conditions followed the recommendations of default settings for the SNP assay, except that the number of cycles were set to 55 and run at $92^{\circ} \mathrm{C}$ for $15 \mathrm{~s}$. The ramp speed was set to standard. A post-PCR read was done for allelic discrimination using appropriate software (SDS version 2.0.4) supplied with the instrument. To test for genotyping errors we used water as negative controls and re-genotyped randomly chosen samples to confirm the results. Case-control analysis between individual sequence variants in MIRO1 and MIRO2 were performed using a Chi-square $\left(\chi^{2}\right)$ test [43]. Three PD patients previously known to carry the pathogenic G2019S mutation in leucine-rich repeat kinase 2 (LRRK2) were excluded from the association analysis. Distribution of genotypes in controls was tested for consistency with the Hardy-Weinberg equilibrium. Statistical significance was defined as $p<0.05$.

Table 1. Investigated Polymorphisms in MIRO1 and MIRO2, their Nucleotide Position, Consequence, Genomic Location and Functional Protein Region

\begin{tabular}{|c|c|c|c|c|}
\hline Reference Sequence & Nucleotide Position and Change & Amino Acid Change & Genomic Location & Protein Domain \\
\hline rs 28630420 & c. $766 \mathrm{~A}>\mathrm{T}$ & Thr256Ser & Exon 11 & EF-hand \\
\hline rs34538349 & c.1048_1049insT & Cys350Leu & Exon 13 & EF-hand \\
\hline rs1139897 & c. $734 \mathrm{G}>\mathrm{A}$ & Arg245Gln & Exon 10 & EF-hand \\
\hline rs3743912 & c. $708 \mathrm{C}>\mathrm{T}$ & Asn236Asn & Exon 10 & EF-hand \\
\hline
\end{tabular}




\section{RESULTS}

The results from the TaqMan genotyping of the five SNPs in MIRO1 and MIRO2 are presented in Table 2. We did not find any genotypic or allelic (data available upon request) association of the MIRO1 or MIRO2 SNPs screened in our Swedish Parkinson case-control material. The observed frequencies of the controls were in agreement with the Hardy-Weinberg equilibrium (data not shown). For two of the SNPs in MIRO1 (rs28630420, rs34538349) we only observed the wild-type genotype.

To investigate if any of the SNPs had a possible effect on age of onset we stratified the material into early disease onset ( $\leq 50$ years) or late disease onset ( $>50$ years) and compared genotype and allele frequencies. However, such stratification did not reveal any significant associations with disease at genotype or allele (data available upon request) levels, as shown in Table $\mathbf{3}$.

\section{DISCUSSION}

Dysfunctional mitochondria may be the cause or the consequence of DA neuron degeneration in PD. This is the first genetic study analyzing possible links between the two GTPases MIRO1 and MIRO2 and disease in general and neurodegenerative disease in particular. Two functionally important regions in the two Miro proteins are the two GTPase domains and two calcium binding EF-hands [36,37]. Another protein with a GTPase domain linked to PD is leucine- rich repeat kinase 2 (LRRK2), a large protein with multiple domains, including functional Roc GTPase and a protein kinase domain. Several mutations in the kinase domain of LRRK2 are associated with both sporadic and familial PD [44]. Recently, a new mutation (N1437S) localized within the GTPase domain of LRRK2 (PARK8) was found to cosegregate with PD [45]. Furthermore, it has been suggested that the GTPase domain may contribute to the toxicity of LRRK2 [46]. Interestingly, knockdown of 1rk-1, the ortholog of LRRK2 in C. elegans, has been reported to lead to reduced survival and is associated with dysfunctional mitochondria [47]. There are no reports on proteins with EFhand domains being linked to PD today.

It has been shown that all domains of Gem1P (i.e. yeast Miro) are needed for proper mitochondrial morphology [37]. Loss-of-function mutations in the Miro GTPase in Drosophila melanogaster lead to impaired locomotion and premature death of the flies [48]. Based on these findings we selected polymorphisms that are located in functional regions in MIRO1 and MIRO2 to analyze possible association with risk for neurodegeneration. The three selected MIRO1 SNPs are localized in one of the GTPase domains (rs16967164) and in each of the two EF-hands (rs28630420, rs34538349). The two SNPs in MIRO2 (rs1139897 and rs3743912) are situated in one of the EF-hand domains. We did not find association with any of the selected SNPs in our Swedish casecontrol material. For two of the SNPs in MIRO1 (rs28630420 and rs34538349) we only detected the wild-

Table 2. Genotype Frequencies of MIRO1 and MIRO2 Variants in Swedish Patients with Parkinson's Disease (PD) Compared to Matched Neurologically Healthy Controls Analyzed using a Two-sided Chi-square $\left(\chi^{2}\right)$ test

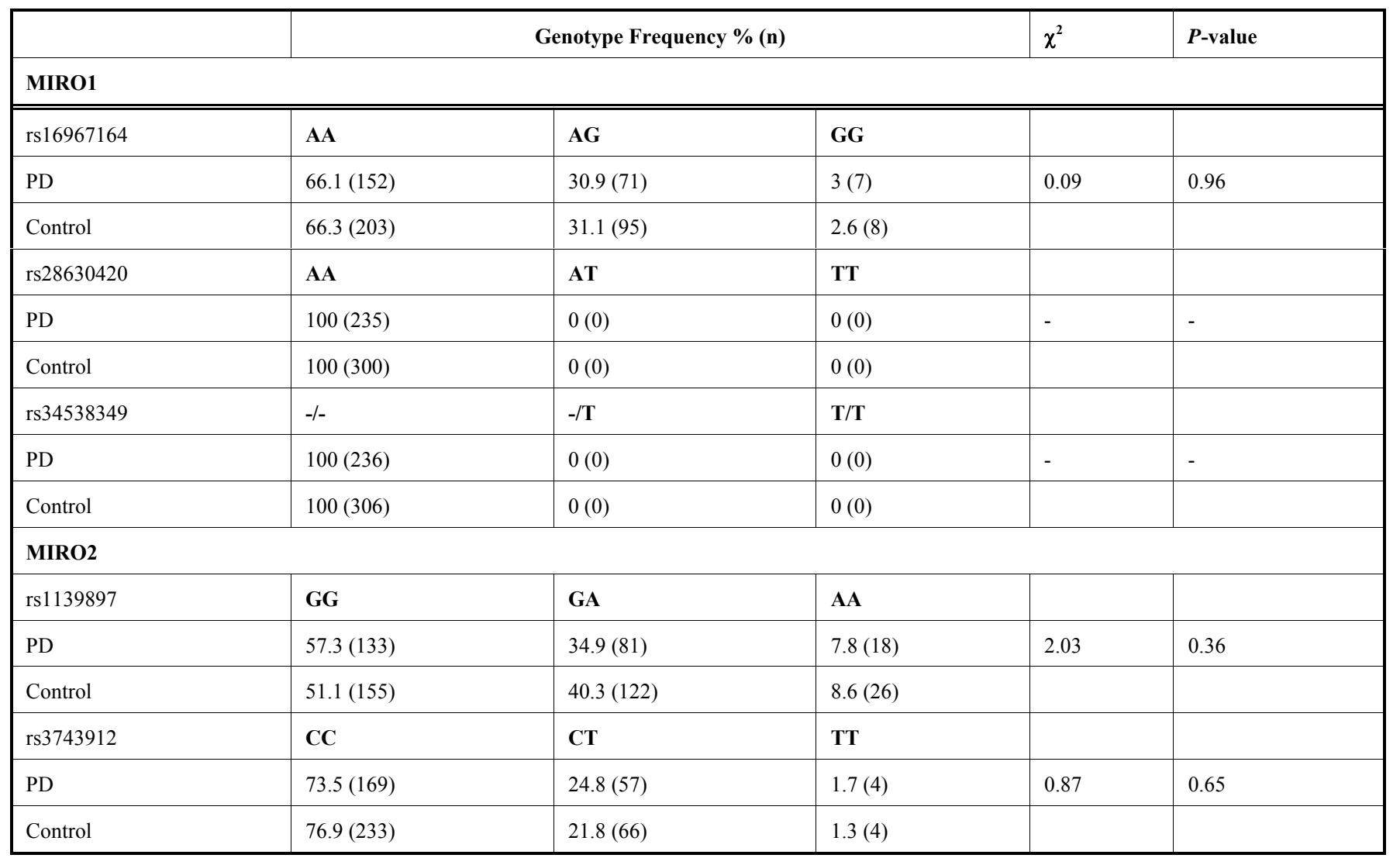


Table 3. Stratification of Parkinson's Disease (PD) Cases into Early ( $\leq 50$ years) and Late Onset ( $>50$ years), Comparing MIRO1 and MIRO2 Genotype Frequencies Analyzed using a Two-sided Chi-square $\left(\chi^{2}\right)$ Test

\begin{tabular}{|l|l|l|l|l|l|l|}
\hline \multicolumn{5}{|c|}{ Genotype Frequency \% (n) } & $\chi^{2}$ & P-value \\
\hline \multicolumn{2}{|l|}{ MIRO1 } & AA & AG & GG & & \\
\hline \hline rs16967164 & $62.3(33)$ & $40.0(18)$ & $3.8(2)$ & 0.45 & 0.80 \\
\hline$\leq 50$ PD & $66.7(118)$ & $31.1(55)$ & $2.2(4)$ & 0.06 & 0.97 \\
\hline$>50$ PD & $66.3(203)$ & $31.1(95)$ & $2.6(8)$ & & \\
\hline Controls & \multicolumn{3}{|l|}{} & AA & & \\
\hline MIRO2 & GG & GA & $5.7(3)$ & 0.79 & 0.67 \\
\hline rs1139897 & $49.1(26)$ & $45.3(24)$ & $8.4(15)$ & 3.51 & 0.17 \\
\hline$\leq 50$ PD & $59.6(106)$ & $32.0(57)$ & $8.6(26)$ & & \\
\hline$>50$ PD & $51.1(155)$ & $40.3(122)$ & TT & & \\
\hline Controls & CC & CT & 0 & - & - \\
\hline rs3743912 & $77.4(41)$ & $22.6(12)$ & $2.3(4)$ & 1.63 & 0.44 \\
\hline$\leq 50$ PD & $72.2(127)$ & $25.6(45)$ & $1.3(4)$ & & \\
\hline$>50$ PD & $76.9(233)$ & $21.8(66)$ & & & \\
\hline Controls & & & & & \\
\hline
\end{tabular}

type genotype in our material of cases and controls. Thus these two mutations tend to be very rare in the area from which our DNA samples have been collected. The results after stratification of the material regarding age of onset did not reveal any age-of-onset association with any of the detected polymorphisms. Further genetic analysis and functional studies in disease are however needed before one can exclude MIRO1 and MIRO2 as potential candidate genes for PD.

\section{CONCLUSION}

Genetic variation in MIRO1 and MIRO2 might influence transport of mitochondria along microtubules, leading to reduced local energy production which can lead to degeneration of DA neurons. Although we did not find a significant association with $\mathrm{PD}$ and the selected polymorphisms in MIRO1 and MIRO2 in our Swedish case-control material, we cannot exclude these Rho GTPases as candidate genes for PD or other neurodegenerative disorders.

\section{CONFLICT OF INTEREST}

None declared.

\section{ACKNOWLEDGEMENT}

We would like to thank Professor Laura Fratiglioni for providing us with control samples from the Swedish National Study on Aging and Care in Kungsholmen (SNAC-K) project and Doctor Fengqing Xiang for excellent technical assistance. The study was supported by the Swedish Research Council, the Swedish Brain Foundation, Karolinska Institutet Funds, Magnus Bergvalls stiftelse, the Swedish
Parkinson Foundation, Åke Wibergs stiftelse and Swedish Brain Power.

\section{REFERENCES}

[1] Kovari E, Horvath J, Bouras C. Neuropathology of Lewy body disorders. Brain Res Bull 2009; 80: 203-10.

[2] Lees AJ, Hardy J, Revesz T. Parkinson's disease. Lancet 2009; 373: 2055-66.

[3] Braak H, de Vos RA, Bohl J, Del TK. Gastric alpha-synuclein immunoreactive inclusions in Meissner's and Auerbach's plexuses in cases staged for Parkinson's disease-related brain pathology. Neurosci Lett 2006; 396: 67-72.

[4] Lin MT, Beal MF. Mitochondrial dysfunction and oxidative stress in neurodegenerative diseases. Nature 2006; 443: 787-95.

[5] Schapira AH. Mitochondria in the aetiology and pathogenesis of Parkinson's disease. Lancet Neurol 2008; 7: 97-109.

[6] Vila M, Ramonet D, Perier C. Mitochondrial alterations in Parkinson's disease: new clues. J Neurochem 2008; 107: 317-28.

[7] Bernardi P, Petronilli V, Di LF, Forte M. A mitochondrial perspective on cell death. Trends Biochem Sci 2001; 26: 112-7.

[8] Chan DC. Mitochondria: dynamic organelles in disease, aging, and development. Cell 2006; 125: 1241-52.

[9] David G, Barrett EF. Stimulation-evoked increases in cytosolic $[\mathrm{Ca}(2+)]$ in mouse motor nerve terminals are limited by mitochondrial uptake and are temperature-dependent. J Neurosci 2000; 20: 7290-6.

[10] Bereiter-Hahn J, Voth M. Dynamics of mitochondria in living cells: shape changes, dislocations, fusion, and fission of mitochondria. Microsc Res Tech 1994; 27: 198-219.

[11] Chen H, Chan DC. Critical dependence of neurons on mitochondrial dynamics. Curr Opin Cell Biol 2006; 18: 453-9.

[12] Hollenbeck PJ, Saxton WM. The axonal transport of mitochondria. J Cell Sci 2005; 118: 5411-9.

[13] Kuznetsov AV, Hermann M, Saks V, Hengster P, Margreiter R. The cell-type specificity of mitochondrial dynamics. Int J Biochem Cell Biol 2009; 41: 1928-39.

[14] Chang DT, Honick AS, Reynolds IJ. Mitochondrial trafficking to synapses in cultured primary cortical neurons. J Neurosci 2006; 26: 7035-45. 
[15] Langston JW, Ballard P, Tetrud JW, Irwin I. Chronic Parkinsonism in humans due to a product of meperidine-analog synthesis. Science 1983; 219: 979-80.

[16] Greenamyre JT, MacKenzie G, Peng TI, Stephans SE. Mitochondrial dysfunction in Parkinson's disease. Biochem Soc Symp 1999; 66: 85-97.

[17] van der Walt JM, Nicodemus KK, Martin ER, et al. Mitochondrial polymorphisms significantly reduce the risk of Parkinson disease. Am J Hum Genet 2003; 72: 804-11.

[18] Otaegui D, Paisan C, Saenz A, et al. Mitochondrial polymporphisms in Parkinson's Disease. Neurosci Lett 2004; 370 : 171-4.

[19] Christian BE, Spremulli LL. Evidence for an active role of IF3mt in the initiation of translation in mammalian mitochondria. Biochemistry 2009; 48: 3269-78.

[20] Koc EC, Spremulli LL. Identification of mammalian mitochondrial translational initiation factor 3 and examination of its role in initiation complex formation with natural mRNAs. J Biol Chem 2002; 277: 35541-9

[21] Abahuni N, Gispert S, Bauer P, et al. Mitochondrial translation initiation factor 3 gene polymorphism associated with Parkinson's disease. Neurosci Lett 2007; 414: 126-9.

[22] Anvret A, Ran C, Westerlund M, et al. Possible involvement of a mitochondrial translation initiation factor 3 variant causing decreased mRNA levels in Parkinson's disease. Parkinsons Dis 2010; 2010: 491751.

[23] Behrouz B, Vilarino-Guell C, Heckman MG, et al. Mitochondrial translation initiation factor 3 polymorphism and Parkinson's disease. Neurosci Lett 2010; 486: 228-30.

[24] Kaguni LS. DNA polymerase gamma, the mitochondrial replicase. Annu Rev Biochem 2004; 73: 293-320.

[25] Anvret A, Westerlund M, Sydow O, et al. Variations of the CAG trinucleotide repeat in DNA polymerase gamma (POLG1) is associated with Parkinson's disease in Sweden. Neurosci Lett 2010; 485: $117-20$

[26] Eerola J, Luoma PT, Peuralinna T, et al. POLG1 polyglutamine tract variants associated with Parkinson's disease. Neurosci Lett 2010.

[27] Luoma PT, Eerola J, Ahola S, et al. Mitochondrial DNA polymerase gamma variants in idiopathic sporadic Parkinson disease. Neurology 2007; 69: 1152-9.

[28] Valente EM, Abou-Sleiman PM, Caputo V, et al. Hereditary earlyonset Parkinson's disease caused by mutations in PINK1. Science 2004; 304: 1158-60.

[29] Kitada T, Asakawa S, Hattori N, et al. Mutations in the parkin gene cause autosomal recessive juvenile parkinsonism. Nature 1998; 392: 605-8.

[30] Poole AC, Thomas RE, Andrews LA, et al. The PINK1/Parkin pathway regulates mitochondrial morphology. Proc Natl Acad Sci U S A 2008; 105: 1638-43.

[31] Matsuda N, Sato S, Shiba K, et al. PINK1 stabilized by mitochondrial depolarization recruits Parkin to damaged mitochondria and activates latent Parkin for mitophagy. J Cell Biol 2010; 189: 211-21.

[32] van Duijn CM, Dekker MC, Bonifati V, et al. Park7, a novel locus for autosomal recessive early-onset parkinsonism, on chromosome 1p36. Am J Hum Genet 2001; 69: 629-34.

[33] Thomas KJ, McCoy MK, Blackinton J, et al. DJ-1 acts in parallel to the PINK1/parkin pathway to control mitochondrial function and autophagy. Hum Mol Genet 2010; 20(1): 40-50.

[34] Weihofen A, Thomas KJ, Ostaszewski B, Cookson M, Selkoe DJ. Pink1 forms a multi-protein complex with Miro and Milton, linking Pink1 function to mitochondrial trafficking. Biochemistry 2009.

[35] Macaskill AF, Brickley K, Stephenson FA, Kittler JT. GTPase dependent recruitment of Grif-1 by Miro1 regulates mitochondrial trafficking in hippocampal neurons. Mol Cell Neurosci 2009; 40(3): 301-12.

[36] Fransson S, Ruusala A, Aspenstrom P. The atypical Rho GTPases Miro-1 and Miro-2 have essential roles in mitochondrial trafficking. Biochem Biophys Res Commun 2006; 344: 500-10.

[37] Frederick RL, McCaffery JM, Cunningham KW, Okamoto K, Shaw JM. Yeast Miro GTPase, Gem1p, regulates mitochondrial morphology via a novel pathway. J Cell Biol 2004; 167: 87-98.

[38] Fransson A, Ruusala A, Aspenstrom P. Atypical Rho GTPases have roles in mitochondrial homeostasis and apoptosis. $\mathrm{J}$ Biol Chem 2003; 278: 6495-502.

[39] Lewit-Bentley A, Rety S. EF-hand calcium-binding proteins. Curr Opin Struct Biol 2000; 10: 637-43.

[40] Macaskill AF, Rinholm JE, Twelvetrees AE, et al. Miro1 is a calcium sensor for glutamate receptor-dependent localization of mitochondria at synapses. Neuron 2009; 61: 541-55.

[41] Baron M, Kudin AP, Kunz WS. Mitochondrial dysfunction in neurodegenerative disorders. Biochem Soc Trans 2007; 35: 122831.

[42] Daniel SE, Lees AJ. Parkinson's Disease Society Brain Bank, London: overview and research. J Neural Transm Suppl 1993; 39: 165-72.

[43] Sham PC, Curtis D. Monte Carlo tests for associations between disease and alleles at highly polymorphic loci. Ann Hum Genet 1995; 59: 97-105.

[44] Belin AC, Westerlund M. Parkinson's disease: a genetic perspective. FEBS J 2008; 275: 1377-83.

[45] Aasly JO, Vilarino-Guell C, Dachsel JC, et al. Novel pathogenic LRRK2 p.Asn1437His substitution in familial Parkinson's disease. Mov Disord 2010; 25: 2156-63.

[46] Xiong Y, Coombes CE, Kilaru A, et al. GTPase activity plays a key role in the pathobiology of LRRK2. PLoS Genet 2010; 6: e1000902.

[47] Saha S, Guillily MD, Ferree A, et al. LRRK2 modulates vulnerability to mitochondrial dysfunction in Caenorhabditis elegans. J Neurosci 2009; 29: 9210-8.

[48] Guo X, Macleod GT, Wellington A, et al. The GTPase dMiro is required for axonal transport of mitochondria to Drosophila synapses. Neuron 2005; 47: 379-93. 